\title{
Asiatic acid stabilizes cytoskeletal proteins and prevents TNF- $\alpha$-induced disorganization of cell-cell junctions in human aortic endothelial cells
}

\begin{abstract}
Endothelial hyperpermeability represents an initiating step in early atherosclerosis and it often occurs as a result of endothelial barrier dysfunction. Asiatic acid, a major triterpene isolated from Centella asiatica (L.) Urban, has previously been demonstrated to protect against tumor necrosis factor (TNF)- $\alpha$-induced endothelial barrier dysfunction. The present study aimed to investigate the mechanisms underlying the barrier protective effect of asiatic acid in human aortic endothelial cells (HAECs). The localization of F-actin, diphosphorylated myosin light chain (diphospho-MLC), adherens junctions (AJs) and tight junctions (TJs) was studied using immunocytochemistry techniques and confocal microscopy. Their total protein expressions were examined using western blot analysis. The endothelial permeability was assessed using In Vitro Vascular Permeability Assay kits. In addition, intracellular redistribution of the junctional proteins was evaluated using subcellular fractionation kits. We show that asiatic acid stabilized F-actin and diphospho-MLC at the cell periphery and prevented their rearrangement stimulated by TNF- $\alpha$. However, asiatic acid failed to attenuate cytochalasin D-induced increased permeability. Besides, asiatic acid abrogated TNF- $\alpha$-induced structural reorganization of vascular endothelial (VE)-cadherin and $\beta$-catenin by preserving their reticulum structures at cell-cell contact areas. In addition, asiatic acid also inhibited TNF- $\alpha$ induced redistribution of occludin and zona occludens (ZO)-1 in different subcellular fractions. In conclusion, the barrier-stabilizing effect of asiatic acid might be associated with preservation of AJs and prevention of TJ redistribution caused by TNF- $\alpha$. This study provides evidence to support the potential use of asiatic acid in the prevention of early atherosclerosis, which is initiated by endothelial barrier dysfunction.
\end{abstract}

Keyword: TNF- $\alpha$ Asiatic acid; Anti-hyperpermeability; Actin; Diphosphorylated myosin light chain; Endothelial cell-cell junctions; Human aortic endothelial cells 